\section{Science Signaling}

DIAAS
Chronoregulation by Asparagine Deamidation

Steven J. Weintraub and Benjamin E. Deverman (October 16, 2007)

Science Signaling 2007 (409), re7. [doi: 10.1126/stke.4092007re7]

The following resources related to this article are available online at http://stke.sciencemag.org. This information is current as of November 11, 2014.

Article Tools Visit the online version of this article to access the personalization and article tools:

http://stke.sciencemag.org/content/2007/409/re7

References This article cites 48 articles, 30 of which you can access for free at: http://stke.sciencemag.org/content/2007/409/re7\#BIBL

Glossary Look up definitions for abbreviations and terms found in this article: http://stke.sciencemag.org/cgi/glossarylookup

Permissions Obtain information about reproducing this article: http://www.sciencemag.org/about/permissions.dtl

Science Signaling (ISSN 1937-9145) is published weekly, except the last December, by the American Association for the Advancement of Science, 1200 New York Avenue, NW, Washington, DC 20005. Copyright 2014 by the American Association for the Advancement of Science; all rights reserved. 


\title{
Chronoregulation by Asparagine Deamidation
}

\author{
Steven J. Weintraub ${ }^{1 *}$ and Benjamin E. Deverman ${ }^{2}$
}

(Published 23 October 2007)

\begin{abstract}
Every asparagine in every protein undergoes nonenzymatic deamidation to aspartate or isoaspartate at a rate determined by the surrounding protein structure and cellular environment. Under physiologic conditions, the deamidation half-life of individual asparagines in proteins is proposed to range from less than a day to several centuries. More than 200 proteins have been shown to undergo deamidation to a meaningful degree, and modeling predicts that hundreds more undergo deamidation at rates that have the potential to be of biological consequence. Because deamidation converts an asparagine into an aspartate or isoaspartate, it introduces a negative charge into a protein and results in the isomerization of a residue. Therefore, deamidation has the potential to change protein function. Additionally, deamidation is thought to render some proteins more susceptible to degradation. In most instances in which asparagine deamidation has been identified in vivo, it is involved in pathology. Hence, deamidation has been viewed primarily as a form of protein damage. However, the pervasiveness and evolutionary persistence of these unstable asparagines suggest that they may have a beneficial role. Notably, the change of even a single neighboring amino acid can have a marked effect on the rate of deamidation of an asparagine. Therefore, the underlying rate of deamidation of any asparagine is genetically programmable. This characteristic, combined with the wide range of deamidation rates that can be programmed, imparts to asparagines the potential to serve as molecular timers that regulate protein function and stability.
\end{abstract}

Nonenzymatic Asparagine Deamidation-Purification Artifact or in Vivo Process

The deamidation of an asparagine residue in a eukaryotic cell is a nonenzymatic process that is initiated by a nucleophilic attack of the peptide bond amino group on the asparagine side-chain amide group. This results in the formation of a cyclic succinimide intermediate that is hydrolyzed to either an aspartate or an isoaspartate residue, with a preference for the formation of the latter because of the asymmetry of the succinimide intermediate (Fig. 1). Therefore, deamidation introduces a negative charge into a protein and results in the isomerization of a residue. Glutamine, the other amino acid with an amide side chain, can also undergo spontaneous deamidation, but this

${ }^{1}$ Division of Urology, Department of Cell Biology and Physiology, The Siteman Cancer Center, Washington University School of Medicine, 660 South Euclid Avenue, Campus Box 8242, St. Louis, MO 63110, USA.

2Division of Biology, California Institute of Technology, 1200 East California Boulevard, Pasadena, CA 91125, USA.

*Corresponding author. E-mail: weintraub@wustl.edu

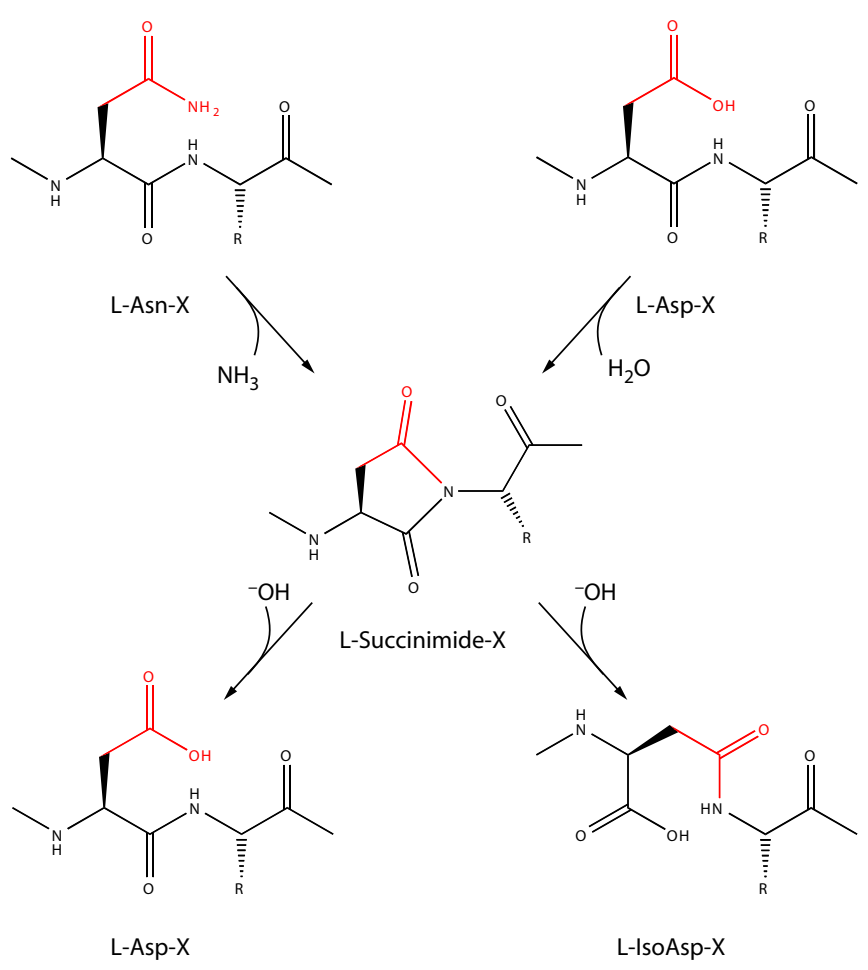

Fig. 1. Deamidation results in the conversion of asparagine to aspartate or isoaspartate. A succinimide intermediate is formed when asparagine is deamidated. The intermediate is then rapidly hydrolyzed to either an aspartate or an isoaspartate. Isoaspartate can also be formed through the isomerization of aspartate.

occurs at a much slower rate than does the deamidation of asparagine (1), so it is likely that nonenzymatic glutamine deamidation is not of the same biological importance as asparagine deamidation. Similarly, aspartate can undergo spontaneous isomerization to isoaspartate (Fig. 1), but this also occurs at a slower rate and at fewer sites than asparagine deamidation (1), so it is not likely to be of the same importance.

It was found that asparagine has a propensity to spontaneously undergo deamidation under mild conditions, at least as early as 1883, by Schulze and Bosshard (2); however, the deamidation of asparagine in proteins was considered nothing more than a purification artifact until the 1960s, when Flatmark and colleagues demonstrated in an elegant series of experiments that cytochrome $\mathrm{c}$ becomes deamidated in vivo $(3,4)$. They first found that there are four electrophoretic variants of monomeric cytochrome $\mathrm{c}$ in bovine heart isolates, which they designated Cy I, Cy II, Cy III, and Cy IV, in order of their decreasing mobility during electrophoresis. They then demonstrated that the major component, Cy I, is spontaneously converted to Cy II, Cy III, and Cy IV in a physiologic buffer. Finally, they found that the 
four variants differ only in the number of amide groups they contain, with Cy II, Cy III, and Cy IV containing fewer amide groups than $\mathrm{Cy} \mathrm{I}$, which indicates that $\mathrm{Cy} \mathrm{I}$ is native cytochrome c, and Cy II, C III, and C IV are deamidated forms of Cy I. Hence, Flatmark had demonstrated that deamidation of cytochrome c occurs spontaneously under physiologic conditions.

Flatmark and his colleague Sletten then sought to determine whether cytochrome c becomes deamidated in vivo (4). They found that the same four variants of cytochrome $\mathrm{c}$ identified in bovine heart are present in multiple organs of the rat. Notably, the relative concentration of each of the variants was the same in all of the organs examined, and the relative concentration of each was the same regardless of whether they extracted the cytochrome $\mathrm{c}$ from tissue using either of two different solvents in which the rate of deamidation would differ (dilute sulfuric acid at $\mathrm{pH} 4.0$ or distilled water). These findings argued strongly that the deamidated forms of cytochrome $\mathrm{c}$ did indeed form in vivo, but Flatmark and Sletten provided conclusive proof of this with a final, particularly elegant experiment (Fig. 2). They first injected rats with ${ }^{59} \mathrm{Fe}$ to label newly synthesized cytochrome c and then, beginning 72 hours later, they administered daily injections of unlabeled iron sorbitol. The unlabeled iron acted to block the incorporation of ${ }^{59} \mathrm{Fe}$ into the cytochrome $\mathrm{c}$ that was synthesized after the first 72 hours. These experiments indicated that ${ }^{59} \mathrm{Fe}$-labeled $\mathrm{Cy}$ I (native form) increased rapidly and then decreased continuously after approximately the first 72 hours. However, as the concentration of ${ }^{59} \mathrm{Fe}-$ labeled $\mathrm{Cy} \mathrm{I}$ decreased, the concentration of ${ }^{59} \mathrm{Fe}-$ labeled $\mathrm{Cy}$ II and Cy III (deamidated forms) continued to increase relative to the amount of ${ }^{59} \mathrm{Fe}-$ labeled Cy I over the 28 days of the study. The simplest explanation for these results is that Cy I was continuously deamidated to Cy II and Cy III in vivo. Had the deamidation of cytochrome c occurred only during the purification process, the ratio of the ${ }^{59} \mathrm{Fe}-$ labeled Cy II and Cy III relative to ${ }^{59} \mathrm{Fe}-$ labeled Cy I would have been the same at all intervals.

\section{The Molecular Clock Hypothesis of Deamidation}

After hearing of Flatmark's studies, a graduate student, Arthur Robinson, questioned why such an exquisitely unstable amino acid as asparagine would be so widely distributed in nature unless its deamidation had a beneficial role (5). Robinson considered Flatmark's finding that each of the deamidation products of native cytochrome $\mathrm{c}$ are present at differing concentrations, but that the relative concentrations of each vary little from tissue to tissue or species to species (4), and postulated that each asparagine in a protein must become deamidated at a rate that is inherent for that particular asparagine. On the basis of this concept, he proposed that asparagine deamidation could play the role of a timer of biological events, with the rate largely set by the primary, secondary, and tertiary structure of the protein surrounding each deamidation site. In this manner, the duration of certain biological processes could be set to occur at a specific rate simply by varying the amino acids that surround asparagine residues within proteins (5). Since then, Robinson, members of his family, and other colleagues have generated experimental evidence to support this molecular clock hypothesis.

In 1970, Robinson and co-workers demonstrated that the rate of asparagine deamidation can indeed be determined by the surrounding amino acid sequence. For example, in a $\mathrm{pH} 7.0$ sodium phosphate buffer at $37^{\circ} \mathrm{C}$, the asparagine in the pentapeptide Gly-Ala-Asn-Ala-Gly has a deamidation half-life of 40 days, whereas the asparagine in the pentapeptide Gly-Arg-Asn-ArgGly has a deamidation half-life of only 11 days (6). This variation in rate is due to both the steric and catalytic effects of neighboring residues (7). Therefore, a change in a neighboring residue can have a profound impact on the rate at which an asparagine becomes deamidated. This makes asparagine deamidation an ideal candidate for a timer that can be genetically programmed to regulate biological events of various durations.

If deamidation does function as a molecular timer with the rate of deamidation set by the neighboring amino acids, it might be expected that asparagine-containing sequences that function as useful timers would be selected for, whereas potentially disruptive sequences (in other words, sequences that undergo deamidation too rapidly) would be selected against.

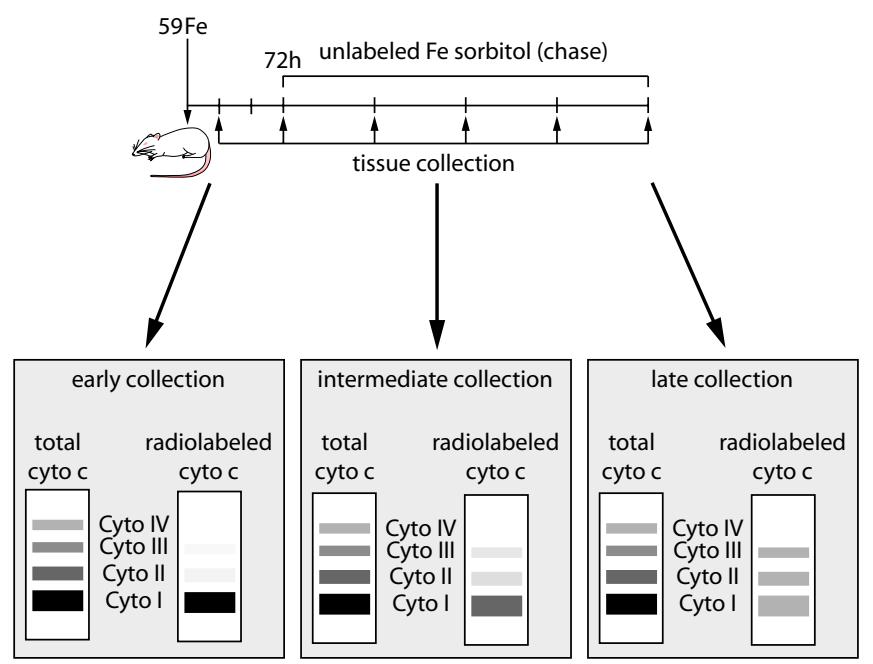

Fig. 2. Deamidation occurs in vivo. Flatmark and Sletten (4) found that there are four forms of monomeric cytochrome $c$ in several bovine and rat tissues. They demonstrated that one of these, Cy I, is the native form of cytochrome $\mathrm{c}$ and that the others-Cy II, Cy III, and Cy IV-are deamidated forms. To determine whether cytochrome $\mathrm{c}$ becomes deamidated in vivo, they first delivered a pulse of ${ }^{59} \mathrm{Fe}$ intravenously to rats, and then, beginning 72 hours later, they administered unlabeled iron sorbitol daily as a chase to block the uptake of ${ }^{59} \mathrm{Fe}$ into newly synthesized cytochrome c. When the cytochrome $c$ was isolated at an early time point, the label was primarily found in Cy I. However, the level of labeled Cy II and Cy III increased relative to the level of labeled Cy I at intermediate and late time points.

Indeed, Robinson and co-workers found that, in a set of 43 proteins, certain amino acids were selected for as neighbors of asparagine and glutamine residues, whereas others were selected against (6). A subsequent analysis of all of the amino acid pairs and triplets in a set of 1465 proteins and peptides revealed that asparagine and glutamine are distributed in proteins with a higher statistical preference for and rejection of certain surrounding sequences than any of the 18 other amino acids (8). These data suggested that there are sequences that contain asparagines that form useful functional units and that these are preferred, whereas other asparagine-containing sequences form disruptive units and are rejected. This is consistent with the hypothesis that certain asparagines and their surrounding sequences function together as molecular timers and 
that only timers with certain deamidation rates can play a useful role in timing biological events. That there are proteins with deamidation half-times of less than a day provides evidence for this hypothesis, as it is likely that such unstable proteins would have been driven to a more stable form by evolutionary pressure if deamidation were merely a form of protein damage.

Because deamidation is irreversible, it would be expected in general that asparagine- and glutamine-containing sequences with short deamidation half-lives would not be found in long-lived proteins. Conversely, deamidation would only be of consequence if it occurred within the lifetime of a protein, and the summation of the deamidation of several amidecontaining residues could serve to time short intervals. A comparison of the total number of asparagines and glutamines in long-lived and short-lived proteins demonstrated a clear inverse correlation between the number of amide-containing amino acids in a protein and the known lifetime of the protein (9). This finding was consistent with a role for asparagine deamidation as an embedded clock that functions during the lifetime of a protein.

It is now clear that in addition to the primary structure, the rate of asparagine deamidation is controlled by the secondary and tertiary structure of the protein (10). A corollary to this is that the rate of deamidation of an asparagine can be altered by a change in secondary or tertiary structure such as might occur if the protein is subject to a posttranslational modification or a change in the interaction with a binding partner (10). Therefore, even though the underlying deamidation rate is set by the protein sequence surrounding the asparagine that undergoes deamidation, the process is subject to multiple levels of control within the protein.

The rate of deamidation is also determined by the cellular environment. For instance, the rate of deamidation of cytochrome $\mathrm{c}$ is altered by changes in $\mathrm{pH}$ - even a small change in $\mathrm{pH}$ within the range of $\mathrm{pH} 7.0$ to 8.0 can alter the rate of cytochrome $\mathrm{c}$ deamidation (3). The rate of deamidation is also determined by the ionic composition and ionic strength of the buffer and the temperature (3). Therefore, the rate of deamidation can be regulated by changes in the cellular environment (Fig. 3).

Robinson and his son, Noah Robinson, devised a computational method for predicting the deamidation half-life of any asparagine in any protein for which the three-dimensional (3D) structure is known $(11,12)$. Their formula combines the observed primary structure-determined deamidation rate with a modifying component that is computed on the basis of the $3 \mathrm{D}$ structure. Using this formula, which has proven to be extraordinarily accurate, they demonstrated that deamidation must be widespread. They first computed the predicted deamidation half-life of 126 human proteins included in the Brookhaven Protein Data Bank and found that $4 \%$ of these proteins would be predicted to have a single-site deamidation half-life of less than 1 day, and $13 \%$ would be predicted to have a single-site deamidation half-life of less than 5 days when incubated in a physiologic phosphate buffer (10). They then computed the predicted deamidation half-lives for all of the asparagines in the 49 Drosophila proteins for which 3D structures were registered in the Protein Data Bank. The deamidation half-lives of these proteins were predicted to range from 1 day to more than 1000 days. Fifteen were predicted to have deamidation half-lives shorter than the intrinsic Drosophila life span of 50 days, and of these, five were predicted to have deamidation half-lives of less than 5 days (13). The findings were similar when they computed the predicted deamidation rates of all 17,935 proteins that were listed in the Protein Data Bank (13). These results predict that deamidation is widespread.

There is also direct evidence that deamidation is widespread. As outlined above, deamidation of asparagine gives rise to aspartate and isoaspartate, with isoaspartate as the major product because of the asymmetry of the succinimide intermediate. The ubiquitously expressed enzyme protein L-isoaspartyl methyltransferase (PIMT) is said to partially "repair" deamidated proteins because it catalyzes the conversion of isoaspartate to aspartate (1). In the PIMT knock-out mouse, isoaspartate abundance at birth throughout most of the tissues is four to eight

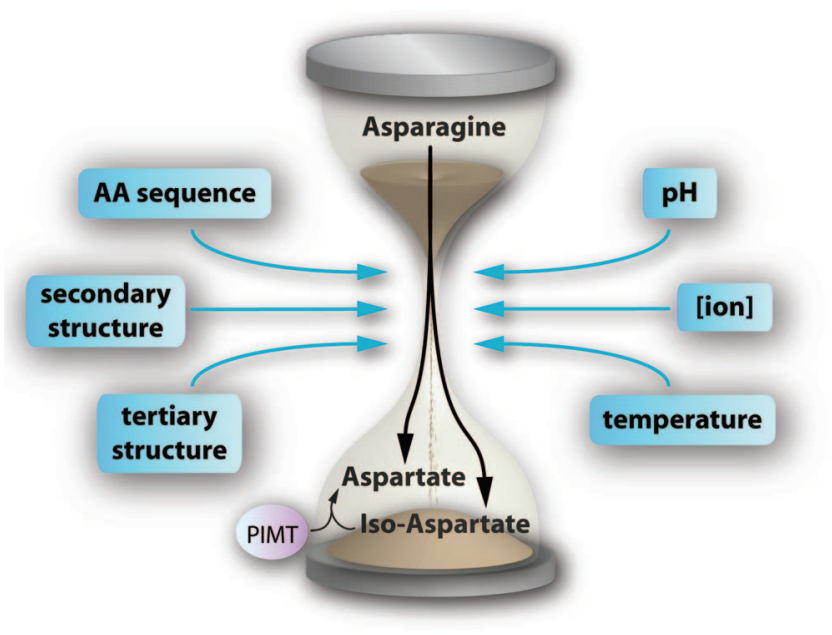

Fig. 3. The rate of deamidation is regulated by multiple factors. The inherent rate of deamidation of an asparagine in a protein is determined by the surrounding sequence (6). However, the rate can be modified by the secondary and tertiary structure of the protein (10), as well as by the $\mathrm{pH}$, ionic composition and strength, and temperature of the environment (3). The enzyme PIMT catalyzes the isomerization of isoaspartate, the major asparagine deamidation product, to aspartate (1).

times its abundance in the tissues of wild-type mice $(14,15)$, which indicates that deamidation occurs ubiquitously even in the tissues of young, otherwise healthy, mice. Because PIMT is expressed in every organism from bacteria to humans, these findings strongly suggest that deamidation is a ubiquitous process in the tissues of a wide range of organisms. Because asparagine deamidation can be genetically suppressed by a single amino acid change in the adjacent sequence, its pervasiveness argues that it must have a beneficial role.

\section{Asparagine Deamidation as a Timer of Protein Turnover}

It was first proposed that deamidation could serve as a timer that determines the life span of a protein because it was found that the deamidated forms of cytochrome c have a more "open" structure, which imparts increased susceptibility to proteolysis $(6,16)$. Indeed, rabbit muscle aldolase undergoes deamidation at a single site that has an in vivo deamidation half-life that is similar to the in vivo half-life of the protein (17). This suggests that deamidation at a single site regulates the protein's turnover. 
Moreover, the rate of deamidation of a pentapeptide that corresponds to the asparagine-containing carboxyl peptide of rabbit muscle aldolase has a deamidation half-life of 6.4 days in a physiologic buffer (18), a rate that is similar to the in vivo deamidation half-life and protein half-life of 8 days. This suggests that the rate of turnover of rabbit muscle aldolase is determined by a molecular timing unit formed by a single asparagine and its surrounding sequence.

Similar amide timing units also regulate cytochrome c turnover, albeit in a more complex manner. By comparing the in vitro deamidation rate of a corresponding peptide, it was determined that the deamidation of the $\mathrm{C}$-terminal sequence

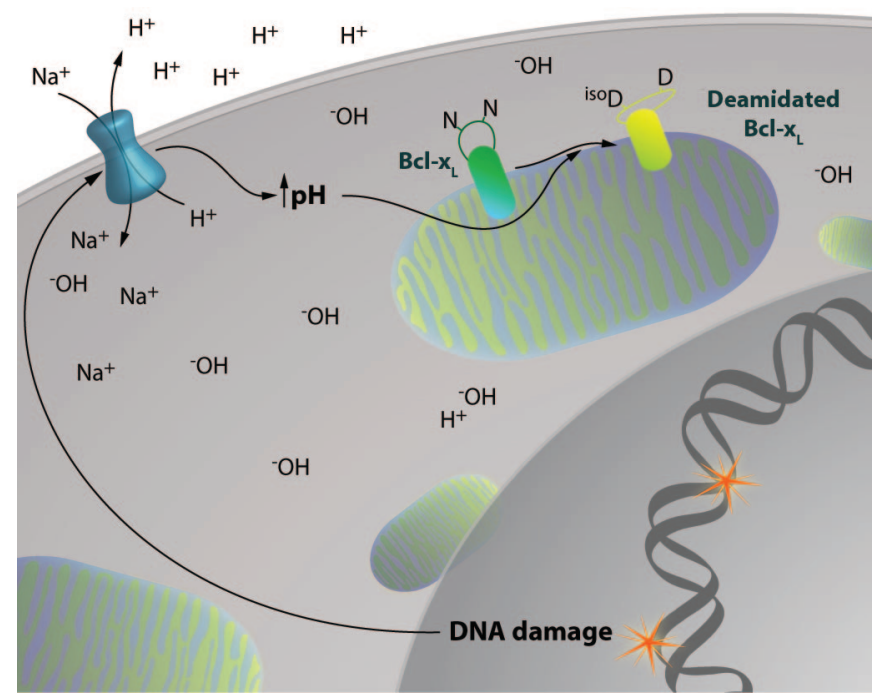

Fig. 4. DNA damage activates NHE1 to alkalinize cytosolic $\mathrm{pH}$, which increases the rate of $\mathrm{Bcl}-\mathrm{x}_{\mathrm{L}}$ deamidation. $\mathrm{Bcl}-\mathrm{x}_{\mathrm{L}}$ is nonenzymatically deamidated at $A s n^{52}$ and $A s n^{66}$. The rate of deamidation is increased in susceptible tumor cells that are treated with DNAdamaging antineoplastic agents (24). Deamidation of $\mathrm{Bcl}-\mathrm{x}_{\mathrm{L}}$ decreases cellular Bcl- $x_{L}$ antiapoptotic activity [(24); see erratum (25)]. The NHE1-mediated early increase in cytosolic pH that occurs in DNA damage-induced and other forms of apoptosis (26-30) mediates the increased rate of $\mathrm{Bcl}-\mathrm{x}_{\mathrm{L}}$ deamidation (31). The mechanism by which DNA damage activates NHE1 is unknown at present.

Thr-Asn-Glu, the first cytochrome c deamidation, is sequencecontrolled. This deamidation apparently leads to a change in the $3 \mathrm{D}$ structure of cytochrome $\mathrm{c}$ that accelerates the rate of the second deamidation. This second deamidation correlates with the onset of cytochrome $\mathrm{c}$ degradation $(4,19,20)$. Therefore, for some proteins, the rate of deamidation correlates with the rate of protein turnover, which suggests that deamidation can function as a timer that regulates turnover. To our knowledge, however, this has never been assessed directly.

\section{Regulation of the Rate of Asparagine Deamidation by Changes in Intracellular $\mathrm{pH}$}

The Bcl-2 family of proteins, which regulates the cellular response to apoptotic stimuli, is composed of both proapoptotic and antiapoptotic proteins (21). There is strong evidence that the antiapoptotic $\mathrm{Bcl}-2$ protein $\mathrm{Bcl}-\mathrm{x}_{\mathrm{L}}$ has a primary role in determining cellular susceptibility to antineoplastic therapy (22). It was first noted by Aritomi and co-workers that $\mathrm{Bcl}-\mathrm{x}_{\mathrm{L}}$ undergoes deamidation in vitro (23). Subsequently, it was found that Bcl- $\mathrm{x}_{\mathrm{L}}$ is deamidated at $\mathrm{Asn}^{52}$ and $\mathrm{Asn}^{66}$ in vivo and that the rate of deamidation of $\mathrm{Bcl}-\mathrm{x}_{\mathrm{L}}$ increases in response to treatment with DNA-damaging antineoplastic agents in susceptible tumor cells (24). Deamidation of Bcl- $\mathrm{x}_{\mathrm{L}}$ increases cellular susceptibility to apoptosis [(24); see erratum (25)]. Because deamidation is a relatively slow process, $\mathrm{Bcl}-\mathrm{x}_{\mathrm{L}}$ deamidation could serve as chronometric buffer that affords the cell time to repair low-level DNA damage before it becomes committed to undergo apoptosis (24).

$\mathrm{Bcl}-\mathrm{x}_{\mathrm{L}}$ is the first known example of a protein in which the rate of deamidation is regulated by cellular signaling. The rate of Bcl- $\mathrm{x}_{\mathrm{L}}$ deamidation increases with increasing $\mathrm{pH}$ (24), and the two $\mathrm{Bcl}-\mathrm{x}_{\mathrm{L}}$ asparagines that become deamidated are predicted to undergo nonenzymatic $\mathrm{Bcl}-\mathrm{x}_{\mathrm{L}}$ deamidation rapidly at $\mathrm{pH} 7.4(11,24)$. Additionally, a small change in $\mathrm{pH}$ between pH 7.0 and 8.0 can substantially increase the rate of deamidation of a protein (3). These findings are notable because there is an increase in $\mathrm{pH}$ to as high as $\mathrm{pH} 8.0$ early in several forms of apoptosis, including DNA damage-induced apoptosis $(25-30)$. The increase in $\mathrm{pH}$ is mediated by the protein $\mathrm{Na}^{+} / \mathrm{H}^{+}$ exchanger 1 (NHE1), a ubiquitously expressed protein that regulates cytosolic $\mathrm{pH}(25-29)$. Together, these findings strongly suggest that the increased rate of $\mathrm{Bcl}-\mathrm{x}_{\mathrm{L}}$ deamidation that occurs in response to DNA damage is stimulated by the NHE1-mediated increase in cytosolic pH (Fig. 4), and indeed, this has recently been confirmed (31). Therefore, asparagine deamidation can serve as a timer that varies in rate in response to changes in the cellular environment. It is likely that alterations in $\mathrm{pH}$ or other solvent properties of the cellular environment regulate the rate of deamidation of other proteins, as well as that of $\mathrm{Bcl}-\mathrm{x}_{\mathrm{L}}$.

\section{Asparagine Deamidation May Serve as Timer of Chromatin Remodeling}

Eukaryotic DNA is packaged into chromatin primarily through its interaction with histone proteins. DNA is wrapped around the core histones to form nucleosomes (32). A linker histone, histone $\mathrm{H} 1$, locks the DNA in position at the nucleosome entry and exit sites to enable the formation of higher-order chromatin structure (33). Posttranslational modifications of histones regulate chromatin structure and function (32); therefore, histones have a primary role in the regulation of gene expression.

There are several subtypes of histone H1 (33). One of these, histone $\mathrm{H} 1^{\circ}$, is primarily found in nonproliferating, terminally differentiated cells (34). In such cells, histones are thought to remodel chromatin to suppress transcription. Histone $\mathrm{H} 1^{\circ}$ exists in liver and brain in two to four subfractions; the relative proportion of each varies with age $(35,36)$. These subfractions are made up of native histone $\mathrm{H} 1^{\circ}$, histone $\mathrm{H} 1^{\circ}$ that is acetylated at the $\mathrm{N}$ terminus, and histone $\mathrm{H}^{\circ}$ that is deamidated at $\mathrm{Asn}^{3}$ and either acetylated or unacetylated at the $\mathrm{N}$ terminus $(35,36)$. The abundance of the deamidated forms of histone $\mathrm{H} 1^{\circ}$ is $1.6-$ to 4 -fold as high in the livers and 8- to 12 -fold as high in the brains of 10-month-old rodents, respectively, compared with those of 20-day-old rodents (36). The authors speculated that increased abundance of the deamidated forms in the older tissue might have a functional role in the chromatin remodeling that suppresses transcription in aging tissue. In this role, deamidation of histone $\mathrm{H}^{\circ}$ would serve as a timedependent switch to decrease transcription in aging cells. 


\section{Asparagine Deamidation as a Timer in the Activation of Cell-Matrix Interactions}

Fibronectin is an extracellular matrix protein that has important roles in cell adhesion, migration, growth, and differentiation (37). Fibronectin contains two highly conserved Gly-Asn-GlyArg-Gly (NGR) loops, which had been suggested to have a role in integrin-mediated cell-fibronectin interactions (38). Curnis and colleagues found that fibronectin aging increases cellfibronectin interactions because of the deamidation of the asparagine in an NGR loop (39). Generation of an isoaspartate at the deamidation site in the NGR loop is necessary to promote the interaction, and consequently, treatment with PIMT, the enzyme that converts isoaspartate to aspartate, decreases adhesiveness. These findings are notable because isoaspartate had previously been thought to have a generalized inactivating function. Thus, Curnis and colleagues identified the first example of activation by isoaspartate formation. They speculated that deamidation of the NGR sequence could act as molecular timer for activating latent integrin-binding sites. It is possible that the NGR loop remains in its inactive form until the fibronectin is secreted from the cell to avoid spurious interactions. It could be activated in a time-dependent manner or it could be kept inactive by PIMT until it is secreted.

\section{Asparagine Deamidation in Disease-Dysregulation of a Normal Process?}

Despite the evidence of its role in regulation of some normal cellular processes, asparagine deamidation continues to be nearly universally viewed as a form of protein damage. This is in part because it is known mostly for its role in pathological conditions. The two most well-studied examples are cataractogenesis (the formation of cataracts) and Alzheimer's disease. Cataractogenesis is thought to be a result of accumulation of insoluble aggregates and cross-linked products of the different forms of crystallins (40). Deamidation of crystallin is one of the most common posttranslational modifications that occur during cataract formation $(41,42)$. Recently, it was demonstrated that crystallins in which asparagines are replaced with aspartates to mimic deamidation aggregate to a much larger extent than do wild-type crystallins and display other characteristics that are associated with cataract formation. These findings suggest an etiologic role for deamidation in cataractogenesis $(43,44)$. Similarly, it is thought that deamidation has a role in development of the neurofibrillary tangles that are pathognomonic for Alzheimer's disease. Neurofibrillary tangles are composed of units called paired helical filaments (45). There is evidence that these develop because of deamidation of the microtubule-binding domain of the tau protein (46). It has been proposed that isoaspartate formation at these sites causes a conformational change that supports self-assembly of the tau protein into paired helical filaments, which suggests that deamidation has a basic role in Alzheimer's disease (47).

Although it is likely that deamidation plays a role in cataractogenesis and Alzheimer's disease, it is not clear that it should, therefore, be viewed simply as a form of protein damage when it is found to contribute to disease. Instead, the deamidation that contributes to certain pathologic conditions may represent the dysregulation of a normally beneficial process. The dysregulation of many beneficial posttranslational modifications results in pathology. One of the clearest examples of this is phosphorylation dysregulation in tumorigenesis. An increase in cyclin- dependent kinase activity and the resultant increased phosphorylation of its effector proteins is required for the generation of many types of tumors (48); however, this increase in phosphorylation is not viewed as a form of protein damage. It could very well be that deamidation has a role in the normal function or regulation of crystallin proteins or the tau protein, but that it becomes dysregulated in cataractogenesis and Alzheimer's disease. It is possible that there is a change in the environment of the protein, such as an increase in $\mathrm{pH}$, that increases the rate of deamidation or there could be a decrease in the clearance of proteins that normally become deamidated. Alternately, there could be a change that alters the manner in which the deamidated molecules interact.

Consistent with the hypothesis that the dysregulation of deamidation leads to pathology are the results of a study on the role of the enzyme PIMT in determining Drosophila life span (49). Ubiquitous overexpression of PIMT in Drosophila has no effect on life span at $25^{\circ} \mathrm{C}$; at $29^{\circ} \mathrm{C}$, however, although the increased temperature shortened the life span of both the control and PIMT-overexpressing Drosophila, flies overexpressing PIMT lived 35\% longer than controls. One possible explanation for this is that, at $29^{\circ} \mathrm{C}$, there is an increased rate of deamidation in both the control and PIMT-overexpressing flies and that overexpression of PIMT can, in part, compensate for the increased isoaspartate formation by catalyzing its conversion to aspartate (49). It is possible that the increase in deamidation at $29^{\circ} \mathrm{C}$ represents the dysregulation of a normally beneficial process.

\section{Conclusion}

Deamidation is a widely occurring posttranslational modification. This is most evident in mice in which the gene for the enzyme PIMT has been knocked out. At birth, these mice have amounts of isoaspartate throughout most of their tissues four to eight times those in the tissues of wild-type mice. Despite its widespread occurrence, asparagine deamidation has remained an obscure and understudied posttranslational modification. Indeed, at the time this Review was written, fewer than 30 references concerned with the physiologic function of asparagine deamidation in eukaryotes were listed in PubMed. This lack of attention is most likely because deamidation has primarily been thought of as a spontaneous, unregulated form of protein damage that is most closely associated with pathological conditions. However, deamidation rates are not unregulated, but genetically programmed, and the programmed rate can be modified through signal transduction pathways, as occurs with DNA damage-induced $\mathrm{Bcl}-\mathrm{x}_{\mathrm{L}}$ deamidation. From the examples cited in this review, it is clear that asparagine deamidation has the potential to regulate several processes; it is possible that deamidation has a role in the regulation of a multitude of processes.

\section{References and Notes}

1. K. J. Reissner, D. W. Aswad, Deamidation and isoaspartate formation in proteins: Unwanted alterations or surreptitious signals? Cell. Mol. Life Sci. 60, 1281-1295 (2003).

2. E. Schulze, E. Bosshard, Über das glutamin. Landwirtsh. Versuchs-Stat. 29, 295-307 (1883).

3. T. Flatmark, On the heterogeneity of beef heart cytochrome c. 3: A kinetic study of the non-enzymic deamidation of the main subfractions (Cy I-Cy III). Acta Chem. Scand. A 20, 1487-1496 (1966).

4. T. Flatmark, K. Sletten, Multiple forms of cytochrome $c$ in the rat: Precursor-product relationship between the main component Cy I and the minor components Cy II and Cy III in vivo. J. Biol. Chem. 243, 1623-1629 (1968).

5. N. E. Robinson, A. B. Robinson, Molecular Clocks: Deamidation of Asparaginyl and Glutaminyl Residues in Peptides and Proteins (Althouse Press, Cave Junction, OR, 2004). 
6. A. B. Robinson, J. H. McKerrow, P. Cary, Controlled deamidation of peptides and proteins: An experimental hazard and a possible biological timer. Proc. Natl. Acad. Sci. U.S.A. 66, 753-757 (1970).

7. N. E. Robinson, A. B. Robinson, Prediction of primary structure deamidation rates of asparaginyl and glutaminyl peptides through steric and catalytic effects. J. Pept. Res. 63, 437-448 (2004).

8. A. B. Robinson, L. R. Robinson, Distribution of glutamine and asparagine residues and their near neighbors in peptides and proteins. Proc. Natl. Acad. Sci. U.S.A. 88, 8880-8884 (1991).

9. A. B. Robinson, Evolution and the distribution of glutaminyl and asparaginyl residues in proteins. Proc. Natl. Acad. Sci. U.S.A. 71, 885-888 (1974)

10. N. E. Robinson, A. B. Robinson, Deamidation of human proteins. Proc. Natl. Acad. Sci. U.S.A. 98, 12409-12413 (2001).

11. N. E. Robinson, Protein deamidation. Proc. Natl. Acad. Sci. U.S.A. 99, 5283-5288 (2002)

12. N. E. Robinson, A. B. Robinson, Prediction of protein deamidation rates from primary and three-dimensional structure. Proc. Natl. Acad. Sci. U.S.A. 98, 4367-4372 (2001)

13. N. E. Robinson, A. B. Robinson, Amide molecular clocks in Drosophila proteins: Potential regulators of aging and other processes. Mech. Ageing Dev. 125, 259-267 (2004).

14. E. Kim, J. D. Lowenson, D. C. MacLaren, S. Clarke, S. G. Young, Deficiency of a protein-repair enzyme results in the accumulation of altered proteins, retardation of growth, and fatal seizures in mice. Proc. Natl. Acad. Sci. U.S.A. 94, 6132-6137 (1997)

15. A. Yamamoto, H. Takagi, D. Kitamura, H. Tatsuoka, H. Nakano, H. Kawano, H. Kuroyanagi, Y. Yahagi, S. Kobayashi, K. Koizumi, T. Sakai, K. Saito, T. Chiba, K. Kawamura, K. Suzuki, T. Watanabe, H. Mori, T. Shirasawa, Deficiency in protein L-isoaspartyl methyltransferase results in a fatal progressive epilepsy. J. Neurosci. 18, 2063-2074 (1998).

16. T. Flatmark, Multiple molecular forms of bovine heart cytochrome c. V. A comparative study of their physicochemical properties and their reactions in biological systems. J. Biol. Chem. 242, 2454-2459 (1967).

17. C. F. Midelfort, A. H. Mehler, Deamidation in vivo of an asparagine residue of rabbit muscle aldolase. Proc. Natl. Acad. Sci. U.S.A. 69, 1816-1819 (1972).

18. J. H. McKerrow, A. B. Robinson, Primary sequence dependence of the deamidation of rabbit muscle aldolase. Science 183, 85 (1974).

19. A. B. Robinson, J. H. McKerrow, M. Legaz, Sequence dependent deamidation rates for model peptides of cytochrome c. Int. J. Pept. Protein Res. 6 31-35 (1974).

20. N. E. Robinson, A. B. Robinson, Molecular clocks. Proc. Natl. Acad. Sci. U.S.A. 98, 944-949 (2001).

21. A. Gross, J. M. McDonnell, S. J. Korsmeyer, BCL-2 family members and the mitochondria in apoptosis. Genes Dev. 13, 1899-1911 (1999).

22. S. A. Amundson, T. G. Myers, D. Scudiero, S. Kitada, J. C. Reed, A. J. Fornace Jr., An informatics approach identifying markers of chemosensitivity in human cancer cell lines. Cancer Res. 60, 6101-6110 (2000).

23. M. Aritomi, N. Kunishima, N. Inohara, Y. Ishibashi, S. Ohta, K. Morikawa, Crystal structure of rat $\mathrm{Bcl}-\mathrm{x}_{\mathrm{L}}$ : Implications for the function of the $\mathrm{Bcl}-2$ protein family. J. Biol. Chem. 272, 27886-27892 (1997).

24. B. E. Deverman, B. L. Cook, S. R. Manson, R. A. Niederhoff, E. M. Langer, I. Rosová, L. A. Kulans, X. Fu, J. S. Weinberg, J. W. Heinecke, K. A. Roth, S. $\mathrm{J}$. Weintraub, $\mathrm{Bcl}-\mathrm{x}_{\mathrm{L}}$ deamidation is a critical switch in the regulation of the response to DNA damage. Cell 111, 51-62 (2002).

25. B. E. Deverman, B. L. Cook, S. R. Manson, R. A. Niederhoff, E. M. Langer, I. Rosová, L. A. Kulans, X. Fu, J. S. Weinberg, J. W. Heinecke, K. A. Roth, S. $\mathrm{J}$. Weintraub, Erratum to $\mathrm{Bcl}-\mathrm{x}_{\mathrm{L}}$ deamidation is a critical switch in the regulation of the response to DNA damage. Cell 115, 503 (2003).

26. L. Huc, L. Sparfel, M. Rissel, M. T. Dimanche-Boitrel, A. Guillouzo, O. Fardel, D. Lagadic-Gossmann, Identification of $\mathrm{Na}^{+} / \mathrm{H}^{+}$exchange as a new target for toxic polycyclic aromatic hydrocarbons. FASEB J. 18, 344-346 (2004).

27. A. R. Khaled, A. N. Moor, A. Li, K. Kim, D. K. Ferris, K. Muegge, R. J. Fisher, L. Fliegel, S. K. Durum, Trophic factor withdrawal: p38 mitogen-activated protein kinase activates $\mathrm{NHE} 1$, which induces intracellular alkalinization. Mol. Cell. Biol. 21, 7545-7557 (2001).

28. N. Tsao, H. Y. Lei, Activation of the $\mathrm{Na}^{+} / \mathrm{H}^{+}$antiporter, $\mathrm{Na}^{+} / \mathrm{HCO}_{3}{ }^{-} / \mathrm{CO}_{3}{ }^{2-}$ cotransporter, or $\mathrm{Cl}^{-} / \mathrm{HCO}_{3}{ }^{-}$exchanger in spontaneous thymocyte apoptosis. J. Immunol. 157, 1107-1116 (1996).
29. W. H. Zhu, T. T. Loh, Effects of $\mathrm{Na}^{+} / \mathrm{H}^{+}$antiport and intracellular $\mathrm{pH}$ in the regulation of HL-60 cell apoptosis. Biochim. Biophys. Acta 1269, 122-128 (1995).

30. H. Y. Dai, N. Tsao, W. C. Leung, H. Y. Lei, Increase of intracellular pH in p53-dependent apoptosis of thymocytes induced by gamma radiation. Radiat. Res. 150, 183-189 (1998)

31. R. Zhao, D. Oxley, T. S. Smith, G. A. Follows, A. R. Green, D. R. Alexander, DNA damage-induced $\mathrm{Bcl}-\mathrm{x}_{\mathrm{L}}$ deamidation is mediated by $\mathrm{NHE}-1$ antiport regulated intracellular pH. PLoS Biol. 5, e1 (2007).

32. T. Kouzarides, Chromatin modifications and their function. Cell 128, 693-705 (2007)

33. J. Zlatanova, P. Caiafa, K. Van Holde, Linker histone binding and displacement: Versatile mechanism for transcriptional regulation. FASEB J.14, 1697-1704 (2000).

34. S. Khochbin, Histone $\mathrm{H} 1$ diversity: Bridging regulatory signals to linker histone function. Gene 271, 1-12 (2001).

35. H. Lindner, B. Sarg, B. Hoertnagl, W. Helliger, The microheterogeneity of the mammalian $\mathrm{H}_{1}{ }^{\circ}$ histone: Evidence for an age-dependent deamidation. J. Biol. Chem. 273, 13324-13330 (1998).

36. H. Lindner, B. Sarg, H. Grunicke, W. Helliger, Age-dependent deamidation of $\mathrm{H} 1^{\circ}$ histones in chromatin of mammalian tissues. J. Cancer Res. Clin. Oncol. 125, 182-186 (1999).

37. R. Pankov, K. M. Yamada, Fibronectin at a glance. J. Cell Sci. 115, 3861-3863 (2002).

38. E. Koivunen, B. Wang, E. Ruoslahti, Isolation of a highly specific ligand for the $\alpha_{5} \beta_{1}$ integrin from a phage display library. J. Cell Biol. 124, 373-380 (1994).

39. F. Curnis, R. Longhi, L. Crippa, A. Cattaneo, E. Dondossola, A. Bachi, A. Corti, Spontaneous formation of L-isoaspartate and gain of function in fibronectin. J. Biol. Chem. 281, 36466-36476 (2006).

40. H. Bloemendal, W. de Jong, R. Jaenicke, N. H. Lubsen, C. Slingsby, A. Tardieu, Ageing and vision: Structure, stability and function of lens crystallins. Prog. Biophys. Mol. Biol. 86, 407-485 (2004).

41. S. R. Hanson, A. Hasan, D. L. Smith, J. B. Smith, The major in vivo modifications of the human water-insoluble lens crystallins are disulfide bonds, deamidation, methionine oxidation and backbone cleavage. Exp. Eye Res. 71, 195-207 (2000).

42. P. A. Wilmarth, S. Tanner, S. Dasari, S. R. Nagalla, M. A. Riviere, V. Bafna, P. A. Pevzner, L. L. David, Age-related changes in human crystallins determined from comparative analysis of post-translational modifications in young and aged lens: Does deamidation contribute to crystallin insolubility? J. Proteome Res. 5, 2554-2566 (2006).

43. R. Gupta, O. P. Srivastava, Deamidation affects structural and functional properties of human $\alpha \mathrm{A}$-crystallin and its oligomerization with $\alpha \mathrm{B}$-crystallin J. Biol. Chem. 279, 44258-44269 (2004).

44. R. Gupta, O. P. Srivastava, Effect of deamidation of asparagine 146 on functional and structural properties of human lens $\alpha \mathrm{B}$-crystallin. Invest. Ophthalmol. Vis. Sci. 45, 206-214 (2004).

45. M. Goedert, M. G. Spillantini, A century of Alzheimer's disease. Science 314, 777-781 (2006)

46. M. Hasegawa, M. Morishima-Kawashima, K. Takio, M. Suzuki, K. Titani, Y. Ihara, Protein sequence and mass spectrometric analyses of tau in the Alzheimer's disease brain. J. Biol. Chem. 267, 17047-17054 (1992).

47. A. Watanabe, K. Takio, Y. Ihara, Deamidation and isoaspartate formation in smeared tau in paired helical filaments: Unusual properties of the microtubule-binding domain of tau. J. Biol. Chem. 274, 7368-7378 (1999).

48. S. J. Elledge, J. Winston, J. W. Harper, A question of balance: The role of cyclin-kinase inhibitors in development and tumorigenesis. Trends Cell Biol. 6, 388-392 (1996).

49. D. A. Chavous, F. R. Jackson, C. M. O'Connor, Extension of the Drosophila lifespan by overexpression of a protein repair methyltransferase. Proc. Natl. Acad. Sci. U.S.A. 98, 14814-14818 (2001).

50. S.J.W. was supported by grants from the N.I.H. and the Elsa U. Pardee Foundation

Citation: S. J. Weintraub, B. E. Deverman, Chronoregulation by asparagine deamidation. Sci. STKE 2007, re7 (2007). 